\title{
Problems with developmental stability in two rodent species from Chornobyl
}

\author{
T.K. Oleksyk ${ }^{1,2}$, M.H. Smith ${ }^{1,2}$, S.P. Gashchak ${ }^{3}$, J.M. Novak ${ }^{1,2}$ and J.R. Purdue ${ }^{4}$ \\ ${ }^{1}$ The University of Georgia's, Savannah River Ecology Laboratory, Drawer E, \\ Aiken, SC 29802, U.S.A. \\ ${ }^{2}$ Institute of Ecology, The University of Georgia, Athens, GA 30602, U.S.A \\ ${ }^{3}$ International Radioecology Laboratory, Slavutych 07100, Ukraine \\ ${ }^{4}$ Illinois State Museum, 1011 East Ash St., Springfield, IL 62703, U.S.A.
}

\begin{abstract}
Changes in developmental patterns are some of the most important effects that may be observed at radioactively contaminated sites like those at Chomobyl. Developmental instability may arise from the interactions between an organism's genotype and its environment and be manifested as deviant morphology. Fluctuating asymmetry (FA) is a measure of deviations from the expected bilateral symmetry of the body. Our objective was to test for differences in FA in two rodent species (Apodemus flavicollis and Clethrionomys glareolus) that live in habitats surrounding the failed Chomobyl reactor. Rodents were collected from four different locations (two contaminated and two reference sites), and a series of adult skulls were photographed and 24 landmarks on each skull were located on digital images from photographed skulls. FA was calculated from the differences in these landmarks on the right and left side of the skull. Significantly more asymmetry $(\sim 2 X)$ was observed in mice and voles living around Chomobyl compared to those living at the uncontaminated reference sites. These are relatively large effects in comparison to those previously found for plants and swallows. FA can be a cheap, easily determined and sensitive indicator of radiation-induced stress in small mammals. FA can be used to prioritize environments for remediation efforts and to efficiently evaluate the effectiveness of the remediation efforts.
\end{abstract}

\section{INTRODUCTION}

Fluctuating asymmetry (FA) is an estimate of small and non-directional departures from the expected bilateral symmetry [10]. FA provides an estimate of developmental stability, because the two sides of a bilaterally symmetrical organism would share the same underlying genotype, and therefore are expected to be identical in a favorable environment $[6,9]$. FA is expected to be higher in populations exposed to significant levels of stress caused by anthropogenic contamination [6]. FA can be estimated as the variance of a distribution among individuals of the differences between the left and right sides.

Environmental radiation has imposed a significant level of stress on populations of urganisms living in the landscapes contaminated after the 1986 Chornobyl meltdown. However, mammalian populations from Chornobyl have not been tested for FA despite the fact that they live in the most contaminated areas around the failed reactor [1]. In the absence of data on humans, small mammal populations can be used as a model for evaluating risks of radioactive contamination in human populations, because they are relatively sensitive to radioactivity.

FA can only be used as a reliable indicator of environmental quality wheñ a substantial number of reference populations with little or no known contamination are sampled. In this case, it is essential to establish the background level of FA in a species using at least two reference populations with no known contamination [7]. In addition, if FA is determined mostly by environmental factors, different species should express similar levels of FA in the same localities if they receive similar amounts of radioactive exposure.

Our overall objective is to determine whether asymmetry is elevated in radioactively contaminated populations compared to that in reference populations of two rodents species 
common around the Chomobyl Nuclear Power Plant. Individual estimates for FA of size and shape of skulls were calculated for each of 3 populations of Apodemus flavicollis and 3 populations of Clethrionomys glareolus. For each of the species we used a highly contaminated population from Chornobyl, and two reference populations located north and south from the radioactive area. We then tested for differences among populations in the amounts of FA.

\section{MATERIALS AND METHODS}

We collected 45 individuals of Apodemus flavicollis and 47 individuals of Clethionomys glareolus from 3 locations in Ukraine (Table 1). The first population, trapped near Gluboke Lake, came from within the highly contaminated $10 \mathrm{~km}$ zone in close proximity to the failed reactor. The captured animals at this site had large amounts of intramuscular contamination by ${ }^{137} \mathrm{Cs}$ [8]. One control population came from a site approximately $350 \mathrm{~km}$ southwest of the failed reactor. This population, Lysychyntsi, had no known radioactive contamination. Another control population, Vyshenky, came from an uncontaminated location $200 \mathrm{~km}$ northeast of Chornobyl.

Our study concentrated on the fluctuating asymmetry (FA) of skulls. Each specimen was cleaned with dermestid beetles and dried. We took pictures of the ventral surface of each skull with a $35 \mathrm{~mm}$ camera using a ring flash to provide even lighting. Two pictures of each skull were taken to account for the effects of placement on the measurement error. Photographs were developed and scanned. Twenty-four evolutionary homologous landmarks were chosen on each side of the skull to represent its entire surface in the same manner for both of the species. Landmarks were independently placed on each of the pictures twice in a digitizing program TPSDIG [12]. All of the statistical analyses were conducted using SAS 8.1 [14].

The $F A$ of size and shape were estimated as described in Klingenberg and McIntyre [3]. Asymmetry in overall size was estimated using centroid size (CS). The CS for each side of each picture for each individual skull was calculated as the square root of the sum of squared distances from a set of landmarks to their centroid [15]. Each skull was scaled to the unit CS in the analysis to eliminate the effect of individual size.

We analyzed the FA of shape by superimposing the configurations of landmarks using the Procrustes method $[3,13]$. First, landmark configurations of the left sides of the skulls are reflected to their mirror images to align corresponding landmarks of right and left sides. After configurations are scaled to unit $\mathrm{CS}$, the points with average coordinates and configurations are rotated around their centroid to achieve the best fit. The output of the Procrustes procedure contains the coordinates of superimposed landmarks. Asymmetry can then be measured as the deviations between the pairs of the corresponding superimposed landmarks.

To estimate the FA of total skull size, we used an ANOVA with CS as the dependent variable, side as a fixed effect and individuals as a random effect as recommended by Palmer [9]. To assess the FA of skull shape, we calculated the overall sums of squares for each of the main effects, interaction term and the error by adding the individual sums of squares for each of the effects across the $\mathrm{X}$ and $\mathrm{Y}$ coordinates [3]. The interaction term in this model represents the variation in left-right differences among individuals, which is a measure of FA. The measurement error was used to test for the significance of the individual*side interaction effect. The variance component of the interaction term provides an unbiased estimate of FA in each population. Degrees of freedom were calculated using the Satterthwaite approximation as suggested by Palmer [9]. Degrees of freedom for the Procrustes ANOVA were the degrees of freedom for each of the effects multiplied by the number of landmark coordinates minus four [3]. Differences in individual population FAs were tested using pairwise $F$-tests. All probabilities 
generated from $F$-tests were tested with a sequential Bonferroni procedure as suggested by Palmer [9].

\section{RESULTS}

Normality of frequency distributions of CS for each population and each species were addressed using Kolmogorov-Smirnov tests of the frequency distribution of the centroid sizes compared to an expected normal distribution. The frequency distributions of our data for each population were inspected for the presence of bimodality (antisymmetry) or unusual outliers. After adjusting the overall error rate to the 0.05 level, there were no significant deviations from normality as indicated by the Kolmogorov-Smirnov test.

Measurement error was addressed during $F$-tests. We were testing whether our FA estimate was significantly larger than predicted due to error alone. In Vyshenky it was not possible to calculate a reliable estimate of the FA of shape compared to the relatively high measurement error for A. flavicollis. We also failed to calculate the significant estimate of FA of shape in $C$. glareolus from Gluboke Lake. All of the other measurements of FA of size and shape were statistically significant $(p \leq 0.05$; Table 1$)$.

FA of size was significantly greater than the variance expected due to the measurement error for all populations except that of $A$. flavicollis in Vyshenky. Thus, this population was not used in the pairwise comparison. FA of size was significantly higher at Gluboke Lake than in the reference populations for $A$. flavicollis (Table 1). FA of shape was not different between populations of $C$. glareolus in Lysychyntsi and Vyshenky. Measurements of FA of size were always higher in populations of $C$. glareolus then in $A$. flavicollis from the same locations.

FA of shape was significantly larger than the variance expected due to measurement error for all the populations. The FAs of shape in Gluboke Lake was significantly greater in FA compared to the other two populations both for A. flavicollis and C. glareolus (Table 1, Fig. 1,2). Measurements of FA of shape also did not differ from each other at the same locations between the two different species $(p \geq 0.05$, Table 1$)$.

\section{DISCUSSION}

Fluctuating asymmetry (FA) refers to the difference between the right and left sides in characters that should otherwise be bilaterally symmetrical. It is likely to be a consequence of epigenetic stress that affects individuals during development. Ionizing radiation should impose stress on individual animals in the Chornobyl contaminated landscapes. We predicted that higher values of FA would be observed in animals of different species of small mammals from the contaminated site closest to the failed reactor as compared to those from the uncontaminated reference sites.

Overall, a higher level of FA was documented for the population in closest proximity to the failed Chornobyl reactor for the asymmetry of both size and shape in two species of small mammals. Fluctuating asymmetry of shape was higher in the population from Gluboke Lake than in two reference populations outside the contaminated site. In turn, reference populations were not different from each other. FA of size was always higher for $C$. glareolus than for $A$. flavicollis. Finally, levels of asymmetry of shape were the same in two different species from the same locations.

FA has been extensively used as a measure of developmental stability because of it's presumed environmental basis. However, the environmental influence of stress on developmental instability and consequently on FA as its measure is not straightforward. Genetic components of FA are generally low, whereas the environmental components are usually high. 
Table 1: Estimated values of the fluctuating asymmetry (FA) of size and shape for two different species of small mammals from a population in Chornobyl (Gluboke Lake) and two reference populations (Lysychyntsi and Vyshenky). Significant values at $\mathbf{p} \leq \mathbf{0 . 0 5}$ are underlined. Degrees of freedom are calculated with Satterthraite procedure. Differences between populations are tested with pairwise $F$-tests. $P$-values for the pairwise comparisons are Bonferroni corrected. Populations that were significantly different in the pairwise FA comparisons are indicated in the last column. Values that are significantly different are marked by different letters ( $F$-test, $\mathrm{A} \neq \mathrm{B}, p \leq 0.05$ ).

\begin{tabular}{llllllllll}
\hline & \multicolumn{3}{c}{ Clethrionomys glareolus } & \multicolumn{5}{c}{ Apodemus flavicollis } \\
\hline FA of Size & $N$ & d.f. & FA values & $\begin{array}{l}\text { Pairwise } \\
\text { Comparisons }\end{array}$ & $N$ & d.f. & FA values & $\begin{array}{l}\text { Pairwise } \\
\text { Comparisons }\end{array}$ \\
\hline 1. Lysychyntsi & 15 & 14 & $\underline{2.159 \times 10^{-07}}$ & A & 15 & 11 & $\underline{1.07 \times 10^{-08}}$ & A \\
2. Gluboke Lake & 12 & 11 & $2.576 \times 10^{-07}$ & not tested & 13 & 7 & $\underline{4.05 \times 10^{-08}}$ & B \\
3. Vyshenky & 20 & 19 & $\underline{1.372 \times 10^{-07}}$ & A & 17 & 1 & $1.49 \times 10^{-08}$ & not tested \\
\hline FA of shape & & & & & & & & \\
\hline 1. Lysychyntsi & 15 & 352 & $\underline{8.766 \times 10^{-08}}$ & A & 15 & 329 & $\underline{7.49 \times 10^{-08}}$ & A \\
2. Gluboke Lake & 12 & 234 & $\underline{1.556 \times 10^{-07}}$ & B & 13 & 366 & $\underline{1.64 \times 10^{-07}}$ & B \\
3. Vyshenky & 20 & 489 & $\underline{7.512 \times 10^{-08}}$ & A & 17 & 457 & $\underline{9.36 \times 10^{-08}}$ & A \\
\hline
\end{tabular}

Similar estimates of FA of shape in two rodent species are consistent with the hypothesis that FA has mostly environmental origins. On the other hand, FA of size was always higher for C. glareolus than $A$. flavicollis. This finding may reflect differences in the specifics of the natural history for these two species. However, what causes the difference to appear in the size asymmetry but not in the shape asymmetry is not clear. Our study demonstrated an almost two fold increase in FA of shape in the contaminated populations from Chornobyl area for two species of small mammals as compared to their reference populations. Many other field studies have linked toxic agents in contaminated areas and demonstrated FA as an indicator of environmental quality. Radiation from Chornobyl affected levels of FA in three species of plants in areas near the Chornobyl Exclusion Zone [5]. Several studies demonstrated plants increase their FAs close to sources of aerial contamination $[2,4]$. In mammals, high heavy metal concentrations were correlated with high FA values in common shrews [11]. Gray seals have also been reported to have higher levels of FA in highly polluted areas versus those in relatively pristine reference areas [16].

In conclusion, FA of skulls in A. flavicollis mice may represent an indicator of the level of radioactive contamination in the animal's environment. Highly contaminated populations of a small mammal species expressed significantly higher levels of FA calculated both as asymmetry of size and shape in two species of small mammals. Fluctuating asymmetry of shape was higher in the population from Gluboke Lake then in two reference populations south and north from the contaminated site. FA of size was always higher for $C$. glareolus than A. flavicollis. Finally, levels of asymmetry of shape were the same in two different species from the same locations. These are relatively large effects in comparison to those previousiy found for plants and swallows. FA can be a cheap, easily determined and sensitive indicator of radiation-induced stress in small mammals. FA can be used to prioritize environments for remediation efforts and to efficiently evaluate the effectiveness of remediation efforts. 


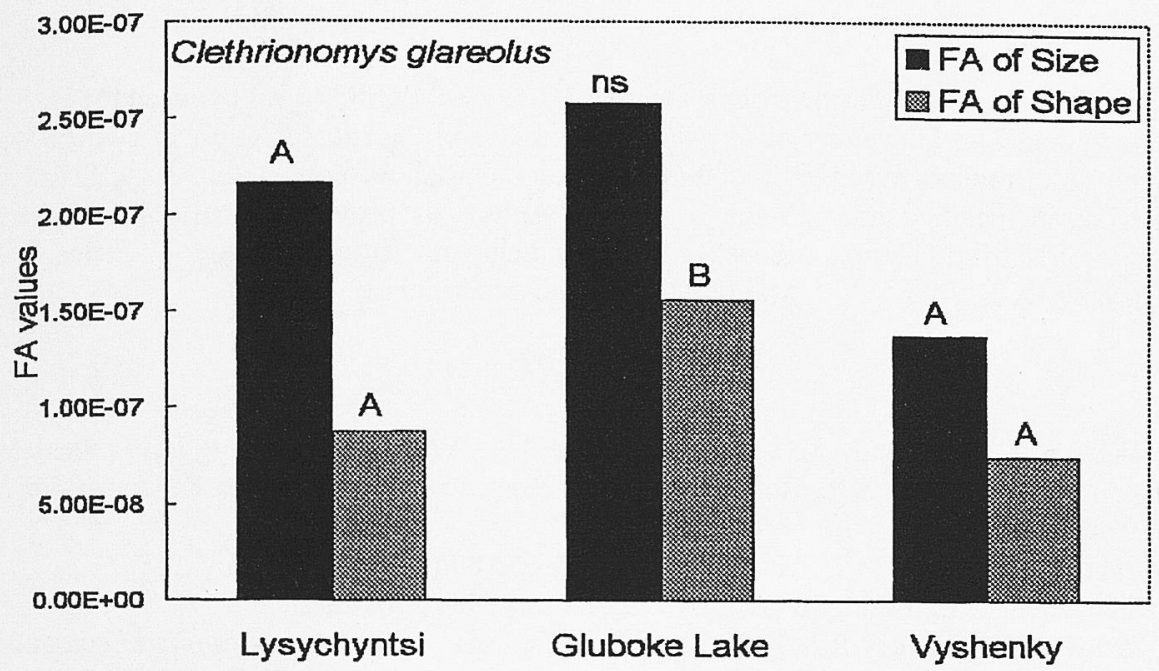

Figure 1: Fluctuating asymmetry (FA) estimates in Clethrionomys glareolus. Values that are significantly different are marked by different letters separately for FA of size and FA of shape. Values that are significantly different are marked by different letters ( $F$-test, $\mathrm{A} \neq \mathrm{B}, p \leq 0.05$ ). Value of $\mathrm{FA}$ of size was not significantly different from zero due to the large amounts of variance other than FA at Gluboke Lake ( $\geq 0.05, \mathrm{~ns})$.

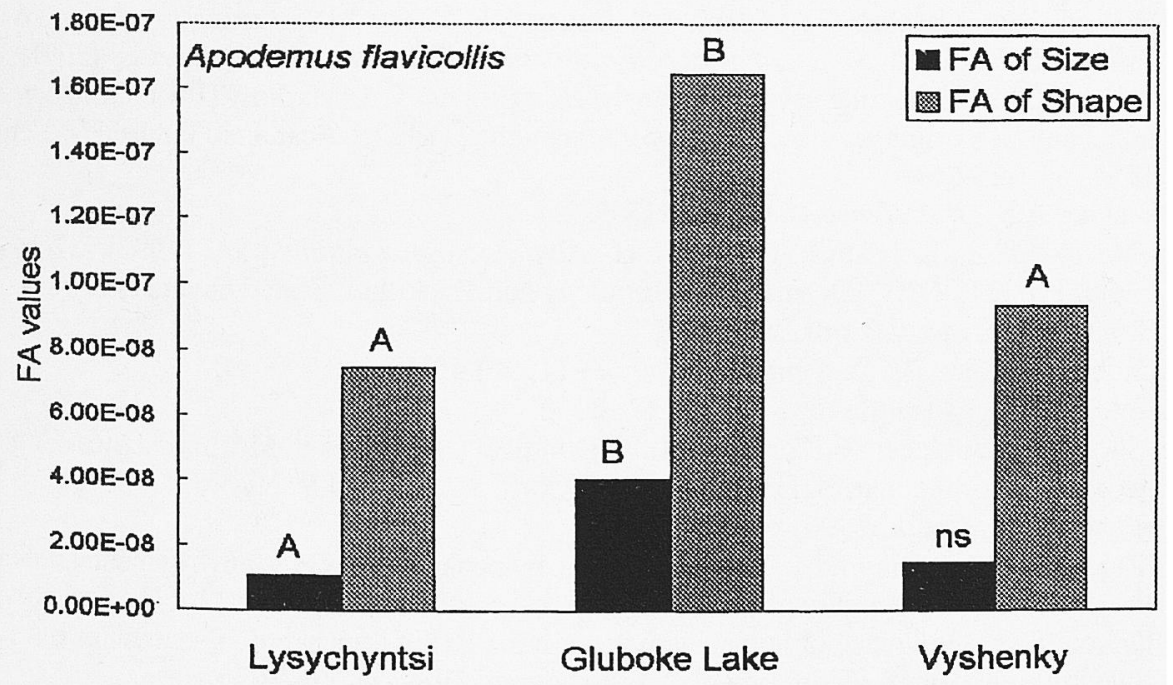

Figure 2: Fluctuating asymmetry (FA) estimates in Apodemus flavicollis. Values that are significantly different are marked by different letters separately for FA of size and FA of shape. Values that are significantly different are marked by different letters ( $F$-test, $A \neq B, p \leq 0.05$ ). Value of FA of size was not significantly different from zero at Vyshenky $(p \geq 0.05, \mathrm{~ns})$. 


\section{Acknowledgements}

Support for this research came from a contract (DE-FC09-96SR18546) between the U. S. Department, of Energy and The University of Georgia. Facilities and logistical support in Ukraine were provided through Dr. Bondarkov, director of the International Radioecology Laboratory, Slavutych, Ukraine. Assistance in handling and storage of rodent skulls was provided by E. McGhee at the Georgia Museum of Natural History, Athens, GA. Other help came from C. Jagoe, T. Glenn, A. Arkhipov, I Chizhevskyj, P. E. Johns, A. J. Majeske, and I. Shchohalevich.

\section{References}

[1] Baker R.J., Hamilton M.J., Van Den Bussche RA., Wiggins L.E., Sugg D.W., Smith M.H., Lomakin M.D., Gaschak S.P., Rudenskaya G.A., Buntova E.G., Chesser R.K., Journal of Mammalogy 77 (1996) 155-170.

[2] Graham J.H., Siegel M.I., Anistranski J., Genetica 89 (1993) 121-137.

[3] Klingenberg C.P., Mclntyre G.S., Evolution 52 (1998) 1363-1375.

[4] Kozlov M.V., Wiseley B.J., Koricheva J.K., Haukoja. E., Journal of Applied Ecology 33 (1996) 1489-1495.

[5] Møller A.P., Oikos 81 (1998) 444-448.

[6] Møller A.P., Swaddle J.P., Asymmetry, Developmental Stability and Evolution, Oxford University Press, Oxford, 1997) $291 \mathrm{pp}$.

[7] Oleksyk T.K., Distribution and effects of Radioactive Contamination in Rodent Populations From Chornobyl. (PhD Dissertation, The Institute of Ecology, The University of Georgia, Athens, GA, 2001) 149 pp.

[8] Oleksyk T.K., Gaschak S.P., Glenn T.C., Jagoe C.H., Peles J.D., Purdue J.R., Tsyusko O.V., Zalissky O.O., Smith M.H., Joumal of Environmental Radioactivity, (in press) (2001).

[9] Palmer A.R., Fluctuating asymmetry analyses: a primer. T.A. Markow (Ed.), Developmental Instability: It's origin and Evolutionary Implications., (Kluver Academic Publishers, Netherlands, 1994) pp. 335-364.

[10]Palmer A.R., Bioscience 46 (1996) 518-532.

[11]Pankakoski E., Koivisto I., Hyvarinen H., Acta Zoologica Fennica 191 (1992) 137-144.

[12]Rohlf F.J., TPSDIG. (Department of Ecology and Evolution, State University of New York at Stony Brook, Stony Brook, NY, 2001.

[13]Rohlf F.J., Slice D., Systematic Zoology 39 (1990) 40-59.

[14] SAS, In, SAS Institute Inc., Cary, N.C., 2000.

[15] Slice D.E., Bookstein F.L., Marcus L.F., RohlfF.J., Advances in Morphometrics. A glossary for geometric morphometrics. L.F. Marcus, M. Corti, A. Loy, G.J.P. Naylor, D. Slice (Eds.), Vol. 284, (Plenum, New York, 1996) pp. 531-551.

[16]Zakharov V.M., Olsson M., Yablokov A.V., Esipenko A.G., Does environmental pollution affect the developmental stability of the baltic grey seal (Halichoerus grypus)? A.V. Yablokov, M. Olsson (Eds.), Influence of human activities on the Baltic ecosystem. Proceesings of the SoviedSwedish Simposium, (Gidrometeoizdat, Leningrad, 1989) pp. 96-108. 